\title{
DIARRÉIA AGUDA EM CRIANÇAS MENORES DE UM ANO: SUBSÍDIOS PARA O DELINEAMENTO DO CUIDAR
}

\author{
Acute Diarrhea in Lesser Children of One Year: \\ Subsidies for the Delineation of Taking Care \\ Diarrea Aguda en Niños Menores de Un Año: \\ Subsidios para el Delineamiento del Cuidado
}

\section{Resumo}

Estudo quantitativo observacional descritivo, teve como fonte de dados o inquérito epidemiológico, para determinar as razões da elevada prevalência de doenças diarréicas agudas em menores de um ano em Ananindeua, Pará. A amostra foi constituída pelas famílias cadastradas no Programa Saúde da Família. Constatou-se que os fatores sócio-econômico-culturais influenciaram na ocorrência de diarréia, pois quanto menores a idade da mãe e a escolaridade maior a prevalência da doença. Esta população vive em área de ocupação, sem saneamento básico, com lixo e dejetos a céu aberto, estando abaixo da linha de pobreza. Quanto ao desmame precoce, observou-se introdução de água e chás nos primeiros 15 dias e de leite artificial no primeiro mês, havendo resistência para utilizar soro oral. Realizavam tratamento caseiro. Conclui-se que, além da reversão dos fatores estruturais, é necessário incluir questões culturais, educativas para uma política pública de cuidar.

Palavras-chave: Enfermagem. Saúde da Criança. Diarréia.

\section{Abstract}

Observational descriptive study with a quantitive approach which data is based on an epidemiological survey aims to determine the reasons of the high prevalence of acute diarrheas illnesses in minors of one year in Ananindeua, Pará. The sample was constituted by the families registered in the cadaster of the Family Health Program. It was evidenced that the social-economic-cultural aspects had influenced in the diarrhea occurrence, therefore how the lesser mother's age and the scholarship, bigger the illness prevalence. This population lives in an occupation area, without basic sanitation, with garbage and dejections in opened sky, being below of the poverty boundary. About precocious weaning, water and tea were introduced in the first fifteen days and artificial milk during the first month, there was a resistance to use oral rehydrating therapy. They used domestic treatment. It concludes that reversion of structural factors, is necessary and cultural matters should be included in educational activities of a public care policy.

\section{Resumen}

El estudio es cuantitativo del tipo observacional descriptivo tuvo como fuente de datos la pesquisa epidemiológica, para determinar las razones del elevado predominio de enfermedades diarreicas agudas en menores de un año en Ananindeua, Pará. La muestra fue constituida por las familias registradas en el Programa Salud de la Familia. Los factores socioeconómicos y culturales determinaron la presencia de diarrea, ya que cuanto menor la edad de la madre y la escolaridad mayor fue el predominio de diarrea. Esta población vive en área de ocupación, sin saneamiento básico, con basura y desechos al aire libre, estando abajo de la línea de pobreza. En cuanto a la precoz suspensión de amamantar, se observa la introducción de agua, en los primeros 15 días y leche artificial en el primer mes, teniendo resistencia para utilizar el suero oral y tratamientos llevados por los vigilantes. Se concluye que además de reversión de los factores estructurales, es necesario incluir cuestiones culturales en las actividades educativas como parte de una política pública de cuidado. 


\section{INTRODUÇÃO}

A doença diarréica aguda (DDA) é uma das doenças mais comuns em crianças em todo o mundo, caracterizando-se pelo aparecimento abrupto. É importante ressaltar que a diarréia atinge pessoas de qualquer faixa etária, mas é na infância que esta afecção causa maior mortalidade. Ela figura como a terceira causa mais comum de doenças em crianças dos países em desenvolvimento e é responsável por cerca de um terço de todas as hospitalizações entre os menores de cinco anos.

Souza ${ }^{1}$ conceitua a diarréia como sendo uma alteração das funções gastrintestinais, que leva à ocorrência de três ou mais evacuações de consistência amolecidas ou líquidas em um período de 24 horas, sendo apontada como uma das afecções que mais ocasiona transtornos à saúde das crianças.

0 Manual de Monitoração da Doença Diarréica (MDDA)² aponta as doenças diarréicas como umas das principais patologias responsáveis pelo desequilíbrio das condiç̃̃es de saúde que caracteriza a população infantil brasileira. Acreditase que o número de casos é bem superior ao divulgado oficialmente, pois há uma deficiência no registro de dados, uma vez que não se trata de uma condição de doença, cuja notificação é compulsória. A notificação eventual associada ao tratamento caseiro gera a subnotificação, mascarando o número/dados das ocorrências de adoecimento por diarréia.

Como problema de saúde pública, a DDA faz parte das principais causas de morbimortalidade infantil. Na atualidade, em escala mundial, ocorrem um bilhão de episódios por ano, causando 3,3 milhões de mortes, de acordo com Sack ${ }^{3}$. A mortalidade infantil destaca-se com uma taxa média mundial de 72 óbitos por mil nascidos vivos (NV), indicando que de cada $10,6 \%$ crianças, uma morre antes de completar um ano, como conseqüência mais grave e imediata da desidratação, segundo Benguigui et a ${ }^{A}$. Entretanto, a persistência da diarréia por um período longo sem tratamento pode evoluir para a desnutrição.

A despeito do progresso na redução da taxa de mortalidade infantil (TMI), em todas as Regiões do Brasil, ainda persistem grandes diferenças inter-regionais. No ano de 1998, a Região Sul apresentava a menor TMl em média de 22,4 por $1.000 \mathrm{NV}$, seguida pela Região Sudeste, em 24,8 por 1.000 NV, e logo depois pelo Centro-Oeste, com 25,1 por 1.000 NV, ficando as Regiões Norte e, principalmente, o Nordeste com as maiores taxas, com uma média de 35,2 e 57,9 por 1.000 NV, respectivamente 5 .

No caso particular do Estado do Pará, o clima quente e úmido ao longo de todo o ano e o maior índice pluviométrico nos meses de dezembro a abril, aliados à inexistência de saneamento básico adequado nesta comunidade, acarretando enchentes, conferem à DDA um certo caráter sazonal neste período.

A Revista de Saúde Pública ${ }^{6}$ descreve que em nosso país a sazonalidade é variável, com aumento na ocorrência de DDA e rotavírus nos meses mais frios ou no período de seca, entre maio e setembro, nos Estados das Regiões Central e Sudeste. Por outro lado, no Norte e Nordeste há ocorrência de rotavírus, distribuída por todo 0 ano.

A DDA, em âmbito nacional, figura em terceiro lugar entre as causas de morbidade infantil em crianças menores de 5 anos, com $30 \%$ dos casos por 1.000 NV, enquanto a infecção respiratória aguda (IRA) figura em segundo lugar, com $40 \%$ por 1.000 NV$^{5}$.

Já no Estado do Pará, observa-se uma relação inversa, levando-se em consideração que a IRA passa a ocupar o segundo lugar, com uma taxa de $10,3 \%$ por $1.000 \mathrm{NV}$, e a DDA o primeiro, com $15,7 \%$ por 1.000 NV. Na comunidade Jardim Nova Vida, também foi observada uma taxa maior de DDA, em torno de $69 \%$ por $1.000 \mathrm{NV}^{7}$, ficando em primeiro lugar, e da IRA, com $57 \%$ por 1.000 (NV), ocupando o segundo lugar. As taxas menores de IRA e DDA do Estado do Pará, comparadas às do Brasil, são justificadas em princípio pela sua notificação, visto que a DDA não é de notificação compulsória, fazendo-se necessário o serviço de monitorização dos casos. A situação acima será ilustrada no Gráfico 1, abaixo.

A pesquisa teve como objetivo geral analisar as causas da elevada prevalência de DDA em crianças menores de um ano da Comunidade Jardim Nova Vida, no Município de Ananindeua, Estado do Pará.

Neste sentido, delineamos como objetivos específicos: a) identificar os fatores associados ao elevado número de casos de DDA em crianças menores de um ano, moradores naquela comunidade; b) traçar o perfil sócio-cultural das famílias com crianças menores de um ano; $c$ ) analisar as implicações dos fatores determinantes na elevada prevalência de DDA em crianças menores de um ano daquela comunidade; d) propor subsídios

Gráfico 1: Dados comparativos de morbidade por DDA e IRA em crianças menores de um ano. Brasil - Pará - CJNV;1999.

$\%$

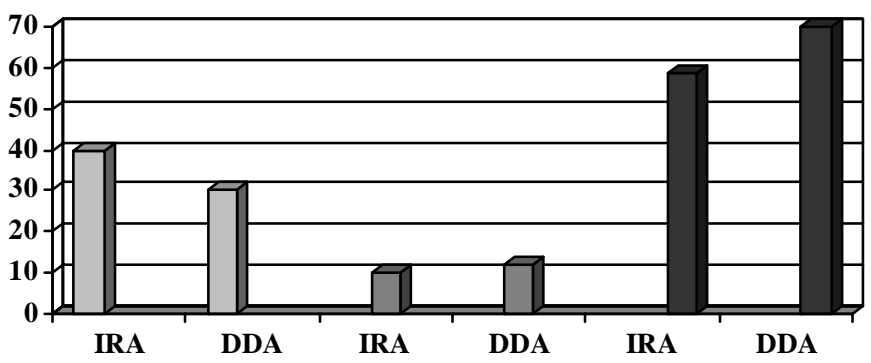

\begin{tabular}{|l|}
\hline$\square$ Brasil \\
$\square$ Pará \\
$\square$ Com. Jardim Nova Vida \\
\hline
\end{tabular}

FONTE: Secretaria Municipal de Saúde de Ananindeua (PA). Sistema de Informação de Atenção Básica. Ananindeua (PA); 1999. 
para o delineamento de políticas de cuidar que minimizem o elevado número de casos de DDA entre aquelas crianças.

Assim, nos apoiamos em Marcondes ${ }^{8}$ para afirmar que os fatores determinantes das DDA vão além do biológico e estão relacionados às condições ambientais (saneamento básico, moradia), nutricionais (padrão alimentar da família), sociais (idade dos pais, grau de instrução), econômicas (renda familiar) e acesso aos serviços de saúde, entre outros. Já o perfil cultural envolve os hábitos adquiridos pela repetição freqüente de um ato, uso ou costume, valores e idéias de um dado grupo social, sendo cumulativos, pois os grupos sociais recebem os elementos culturais vindo de seus antepassados, ao mesmo tempo em que novos elementos podem ser incorporados ao seu patrimônio cultural através do contato com outros grupos.

\section{METODOLOGIA}

Trata-se de um estudo observacional, de cunho descritivo, com abordagem quantitativa.

Segundo Beaglehole ${ }^{9}$, os estudos observacionais permitem que a natureza determine o seu curso sem a intervenção do investigador, que apenas mensura o desfecho. Para Gil ${ }^{10} 0$ objetivo principal destes tipos de estudos é a "descrição das características de determinada população ou fenômeno assim como o estabelecimento de relações entre variáveis (...)"

0 estudo teve como fonte de dados o inquérito epidemiológico, de caráter local, do tipo específico, pois se caracterizou por uma investigação destinada a identificar a situação de uma determinada comunidade diante da DDA, como um agravo à saúde, com o objetivo de conhecer as razões de sua elevada prevalência, distribuição e fatores a ela relacionados.

0 universo da pesquisa é composto por aproximadamente 900 famílias cadastradas no Programa Saúde da Família (PSF), sendo identificadas 136 famílias com crianças menores de um ano, o que corresponde a $100 \%$ das famílias cadastradas no PSF com crianças nesta faixa etária, residentes na comunidade em foco, as quais preenchiam os critérios de inclusão populacional. Estas 136 famílias com crianças menores de um ano correspondem a $8,2 \%$ do total das famílias dessa comunidade, as quais se dispuseram espontaneamente a participar desta pesquisa.

0 instrumento de coleta de dados (questionário) foi aplicado após assinatura do Termo de Consentimento Livre e Esclarecido (TCLE), pelas mães e/ou cuidadores maiores de 18 anos. Procurou-se manter o sigilo e o anonimato, utilizando a ordem de preenchimento dos questionários. Suas participações foram espontâneas e voluntárias, obedecendo a Resolução n 196/1996 (outubro de 1996), que rege sobre os aspectos éticos da pesquisa envolvendo seres humanos. $A$ pesquisa na Comunidade Jardim Novo Vida foi liberada pelo Secretário Municipal de Saúde de Ananindeua-Pará, assim como foi aprovada através de parecer do Comitê de Ética do Núcleo de Medicina Tropical (em anexo).

Quanto aos critérios de inclusão populacional, teve-se como propósito ouvir todas as mães/cuidadores das crianças menores de um ano, cadastradas no PSF e residentes na comunidade. Utilizou-se o termo mães e/ou cuidadores por não ser só a mãe a responsável pela assistência direta à criança.
Foram adotados como critérios de exclusão: crianças maiores de um ano e as menores de um ano com diarréia crônica, de origem metabólica, diagnóstico de intolerância e/ ou alergia alimentar ou por outras patologias associadas.

A variável morbidade para fins de inquérito foi conceituada como a presença de DDA em crianças referidas nos 15 dias anteriores à aplicação do questionário. Entre as variáveis eleitas para o estudo incluem-se as maternas (idade, nível de instrução, situação conjugal, tempo de ausência do domicílio, renda familiar mensal, número de filhos, realização do pré-natal e intervalos interpartais), as relacionadas à criança (idade, peso ao nascer, peso atual, aleitamento materno, alimentação, desmame, diarréia, adoecimento, consumo alimentar durante diarréia, utilização do serviço de saúde e tratamento realizado) e às condições de moradia.

0 Instrumento de Coleta de Dados utilizado foi um questionário com questões abertas e fechadas. 0 procedimento de captação dos sujeitos se deu pela pesquisadora e por quatro aplicadores (alunos de enfermagem, os quais foram selecionados e treinados para aplicação do instrumento), após relação das famílias com crianças menores de um ano liberada pelo coordenador do PSF de Ananindeua. Realizaram-se visitas nos domicílios, sendo esclarecida a importância da pesquisa e da participação das mães/cuidadoras, sendo agendados dia e horário para aplicação do questionário, de acordo com a disponibilidade das mesmas, em suas próprias residências. Os dados obtidos compuseram um Banco de Dados, utilizando-se o programa SPSS for Windows, (Statistical Package for the Social Sciences), versão 8.0., para cálculos das estatísticas necessárias.

Os resultados foram apresentados sob a forma tabular e gráfica. Para compô-los de forma mais homogênea e adequada às análises, utilizaram-se os critérios da Resolução nº 886 da Junta Executiva Central do Conselho Nacional de Estatística, de 26 de outubro de 1966 (Normas Técnicas para Apresentação Tabular da Estatística Brasileira).

Para análise estatística foram utilizados métodos tabulares através do estudo das freqüências absolutas e relativas das distribuições de métodos numéricos através do cálculo de média, desvio-padrão e teste quiquadrado, onde o nível de significância estabelecido foi de 0,05, tendo seu resultado considerado significativo.

\section{APRESENTAÇÃO E DISCUSSÃO DOS RESULTADOS}

O levantamento de dados realizado com as mães/cuidadoras permitiu conhecer a realidade em que vivem as crianças objetos desta pesquisa. Na Tabela 1, na página seguinte, observa-se que $94,9 \%$ das cuidadoras são as mães, destacando-se a ausência da figura masculina voltada para a ajuda no cuidar. A maior faixa etária das cuidadoras está entre 21 e 26 anos, embora exista um percentual bastante relevante $(34,5 \%)$ de mães adolescentes, o que indubitavelmente contribui para 0 aumento do risco de morbimortalidade infantil.

Sob o ponto de vista biológico, a prematuridade, o baixo peso, a má-formação congênita e outros aspectos favorecem o adoecimento de crianças abaixo de um ano. Observa-se, ainda, 
Tabela 1: Distribuição de cuidadores de menores de 1 ano, segundo dados sócio-demográficos. Comunidade Jardim Nova Vida (CJNV) - 2002

\begin{tabular}{|c|c|c|}
\hline VARIÁVEIS & FREQÜÊNCIA & $\%$ \\
\hline \multicolumn{3}{|l|}{ Cuidador } \\
\hline Mãe & 129 & 94,9 \\
\hline Avó & 5 & 3,7 \\
\hline Outros & 2 & 1,4 \\
\hline \multicolumn{3}{|l|}{ Faixa Etária } \\
\hline $15-20$ & 47 & 34,5 \\
\hline $21-26$ & 57 & 41,9 \\
\hline $27-32$ & 21 & 15,4 \\
\hline $33-38$ & 5 & 3,7 \\
\hline+ de 38 & 6 & 4,5 \\
\hline \multicolumn{3}{|c|}{ Situação Conjugal do Cuidador } \\
\hline Com cônjuge (1) & 88 & 64,7 \\
\hline Sem cônjuge (2) & 48 & 35,3 \\
\hline \multicolumn{3}{|l|}{ Renda Familiar Total } \\
\hline Sem fonte de renda & 14 & 10,0 \\
\hline Até $\mathrm{R} \$ 80,00$ & 30 & 22,0 \\
\hline Menos de 1 salário mínimo & 43 & 32,0 \\
\hline 1 salário mínimo & 24 & 17,6 \\
\hline Mais de 1 salário mínimo & 25 & 18,4 \\
\hline \multicolumn{3}{|c|}{ Nível de Instrução dos Cuidadores } \\
\hline Analfabeto total & 7 & 5,1 \\
\hline Analfabeto funcional (3) & 34 & 25,0 \\
\hline Ensino fundamental & 54 & 39,7 \\
\hline Ensino médio & 40 & 29,5 \\
\hline Ensino superior & 1 & 0,7 \\
\hline
\end{tabular}

(1) com cônjuge - os cuidadores que são casados ou vivem maritalmente;

(2) sem cônjuge - os cuidadores solteiros, separados ou viúvos;

(3) analfabeto funcional - pessoas que estudaram até a $4^{\mathrm{a}}$ do ensino fundamental

Obs.: idade das mães $-\mu=24,29$ (média)

$$
\alpha=7,34 \text { (desvio-padrão) }
$$

renda familiar $-\mu=1$ salário mínimo

FONTE: Levantamento de dados realizados na CJNV, Ananindeua, Pará, nov. 2002

que as cuidadoras têm pouca ou nenhuma experiência para identificar os sinais e sintomas da patologia e agravo; pouca habilidade para o cuidar; muitas das vezes são primíparas e com gravidez não planejada/indesejada. Algumas são mães solteiras, com imaturidade emocional; $35,3 \%$ das cuidadoras não têm companheiros, o que influencia negativamente para o orçamento familiar e também para ajuda ao cuidar da criança.

Também se verificou que $32 \%$ dessas famílias vivem abaixo da linha de pobreza. 0 grau de instrução foi considerado baixo, pois $30,1 \%$ pertencem ao grupo de analfabetismo total ou funcional (pessoas com até a $4^{\mathrm{a}}$ série incompleta). Esta situação é considerada um fator de risco para o adoecimento por DDA, pela dificuldade de entender as atividades educativas, muitas das vezes limitando a busca dos benefícios necessários em função da pouca ou nenhuma experiência, levando também à ineficácia do cuidar (higiene e alimentação). Indiretamente, o baixo nível de escolaridade também influencia o desemprego das mães/cuidadoras, elevando, assim, à desigualdade sócio-econômica (Tabela 2).
Tabela 2: Distribuição dos domicílios segundo condições sanitárias - CJNV - 2002.

\begin{tabular}{lcc}
\hline VARIÁVEIS & FREQÜÊNCIA & $\%$ \\
\hline Procedência da água & & \\
Poço a céu aberto & 57 & 41,9 \\
Poço tubular/artesiano & 40 & 29,6 \\
Rede pública/encanada & 21 & 15,3 \\
Ligação clandestina/improvisada (1) & 18 & 13,2 \\
Destino do Lixo & 136 & 100,0 \\
Coleta regular/periódica & & \\
Lançado a céu aberto/rio & 74 & 54,4 \\
Queimado & 31 & 22,8 \\
Enterrado & 30 & 22,1 \\
Destino dos dejetos & 1 & 0,7 \\
Fossa séptica/asséptica & & \\
Céu aberto/sumidouros & 85 & 62,5 \\
Rede pública & 51 & 37,5 \\
Instalação sanitária & 4 & 2,9 \\
Unifamiliar sem água & & \\
Unifamiliar com água & 71 & 51,1 \\
Coletiva sem água & 46 & 32,4 \\
Coletiva com água & 17 & 12,5 \\
\hline
\end{tabular}

(1) Abastecimento através de mangueiras (extensão)

FONTE: Levantamento de dados realizados na CJNV, Ananindeua, Pará, nov. 2002

Quanto ao aleitamento materno, sugere-se que deve ser implementado ainda na sala do parto, sob o regime de livre demanda e sem horário fixo. Para surpresa nossa, quase $60 \%$ das 136 crianças receberam o leite materno após o nascimento, porém, no momento da entrevista, mais de $75 \%$ faziam uso de alimentação mista e artificial.

Em relação ao saneamento básico constante na Tabela 2, pode-se observar que os domicílios possuem cobertura insatisfatória da rede pública de água e não dispõe de acesso ao serviço de esgoto sanitário (Figura 1).

Figura 1: Fossa asséptica (sumidouros).

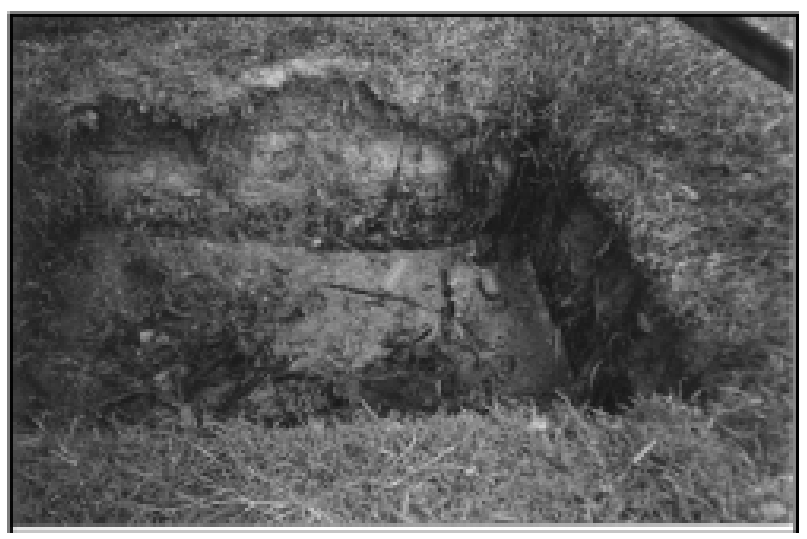

Foto: Ivonete Pereira

Aproximadamente um terço desta população recorre a poço a céu aberto, estando este, em alguns domicílios, sem proteção e ao mesmo nível do sanitário e com uma distância de aproximadamente 5 metros (Figura 2), cuja exposição ao 
ambiente contribui com o agravo à saúde, aumentando a possibilidade de ocorrência de casos de DDA.

Figura 2: Poço a céu aberto.

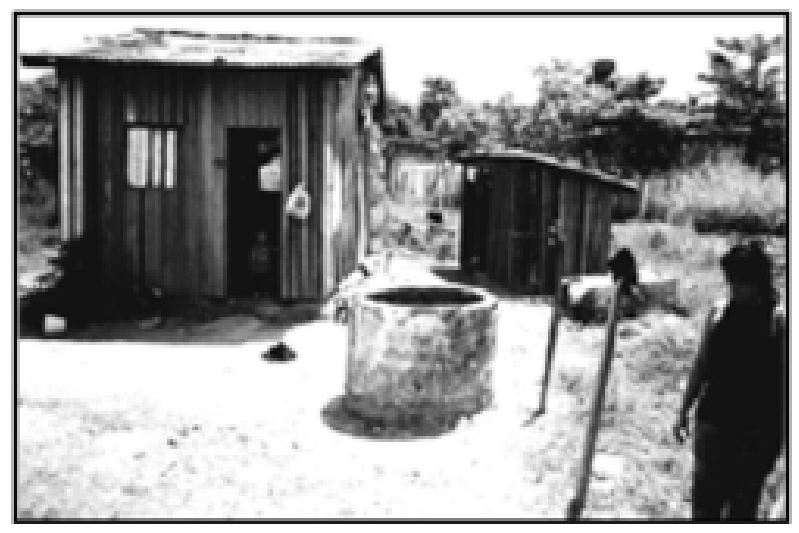

Foto: Ivonete Pereira

Para Silva ${ }^{10}$, os poços só devem ser cavados em locais adequados, com uma probabilidade de um bom suprimento de água subterrâneo limpa, geralmente com 10 a 15 metros de profundidade, quando cavados a mão. Especifica ainda que a metragem correta para construir uma fossa seca é de no mínimo 15 metros de distância da fossa para o poço, dependendo das condições do terreno, não podendo o terreno declinar na direção da fossa para o poço.

Cabe ressaltar que, ainda assim, em alguns domicílios as ligações de água e esgoto ocorrem de maneira clandestina (através de mangueira), sem o devido cuidado higiênico e em condições insatisfatórias, possibilitando a contaminação da rede de água, podendo até ocorrer contato com os dejetos, conforme demonstramos na Figura 3.

Figura 3: Abastecimento de água com ligação clandestina ("gato") da CJNV.

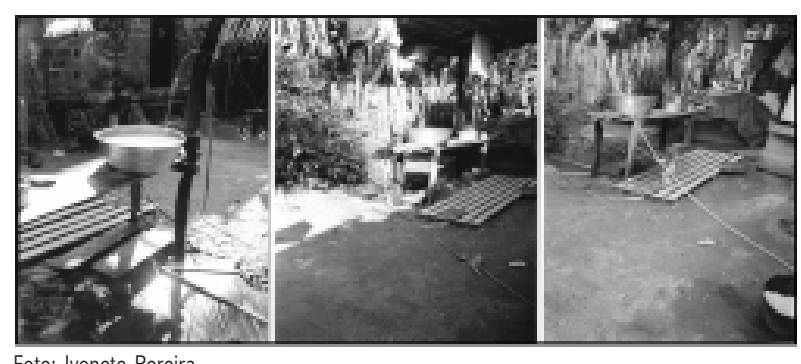

Foto: Ivonete Pereira

\section{CONSIDERAÇÕES FINAIS}

0 elevado número de casos de DDA identificados em nosso estudo está diretamente relacionado com os dados sócioeconômico-culturais, das condições

ambientais e de infra-estrutura.

Além disso, temos de considerar que o fato de a mulher ser pobre, em fase de procriação, com baixa renda familiar, somado às condições ambientais e de infra-estrutura, é comprometedor para a melhoria das condições de saúde, considerando que estas famílias vivem em uma área de ocupação, sem as mínimas condições de habitabilidade.

Ao determinarmos o perfil sócio-cultural das famílias com crianças menores de um ano, procurou-se analisar as implicações desse fator na elevada prevalência de DDA nessas crianças. Encontrou-se nas condições neonatais um número bastante relevante de mães que realizaram o pré-natal $(93,4 \%)$, porém o número de consultas se tornou insuficiente de acordo com o preconizado pelo Ministério da Saúde (MS), que seria no mínimo de 5 consultas; a nossa média foi de 2 consultas. Somado a isto, 0 baixo peso ao nascer com $13,8 \%$, torna-se um risco maior de adoecimento por DDA, tendo sido apontado como o principal determinante para a possibilidade de sobrevida de um recémnascido no período neonato e mesmo no primeiro ano de vida. Considerando-se, ainda, que as crianças com baixo peso apresentam risco de morte de 22,6 vezes maior do que as de peso adequado, as de baixo com DDA têm o risco aumento para 25 vezes. No caso da comunidade em estudo, este risco torna-se maior uma vez que 0 fator de proteção está deficitário, considerando a elevada taxa $(76,5 \%)$ de desmame precocemente.

As implicações desses fatores no adoecimento das crianças menores de um ano por DDA, na Comunidade Jardim Nova Vida (CJNV), são também reflexos das condições de saneamento deficitárias da ocupação, da baixa escolaridade e idade das cuidadoras, e a influência cultural marcante na comunidade reflete-se na falta de busca aos serviços de saúde, e, por conseguinte, na menor utilização do soro oral. Todos estes fatores estão aliados, também, à diversidade da herança cultural nortista, dando origem a variações de comportamentos de saúde-doença pelos diversos segmentos de nossa sociedade.

Partindo-se do princípio que cada comunidade adquire seus próprios conhecimentos, crenças, atitudes, práticas de cuidados com as crianças, com a alimentação e com a doença, não é possível seguir uma única regra universal para a assistência. Por isso, é importante considerarmos que a elevação do padrão da assistência à saúde depende do profissional enfermeiro atuar diretamente na comunidade, conhecendo seus valores culturais, tomando conhecimento e compreendendo melhor o meio de vida da comunidade, para que possa desenvolver alternativas culturalmente aceitáveis pelas cuidadoras.

Em nosso estudo, a cultura de mães/cuidadoras de crianças menores de um ano da CJNV reflete-se nos tipos de alimentos selecionados por elas (carimã, farinha de mandioca etc.), na forma como são preparados (mal cozidos, gordurosos), nas crenças de que o leite materno é fraco, insuficiente, e que a mama está seca, que a criança com diarréia tem quebranto e mal olhado, além de questões relacionadas à dentição.

Ao encerrarmos este trabalho, aumenta a nossa responsabilidade, na medida em que temos consciência de que é preciso mudar, inovar e avançar nas questões referentes à prevenção das doenças, para que possamos proporcionar mudanças no relacionamento dos profissionais de saúde, em particular dos enfermeiros, que atendem direta e permanentemente as mães e/ou cuidadoras. Há necessidade de que a comunidade seja envolvida, não apenas como alvo de informação, mas repartindo com ela a responsabilidade de buscar alternativas por um eficaz trabalho preventivo, através do conhecimento de seus valores culturais, em especial aqueles relacionados à saúde da criança.

Diante disso, propomos as seguintes recomendações: aprimoramento do Sistema de Vigilância, no controle das DDA, desenvolvimento de políticas públicas de saúde voltadas para 
áreas de ocupação, priorizando o atendimento à criança; operacionalização dos serviços, recursos e sistemas de informações já existentes; identificação e monitoramento das crianças com diarréia e risco de agravamento; ampliação de campanha de divulgação das ações de saúde; realização de treinamentos e capacitações sistemáticas dos profissionais de saúde sobre os programas existentes, que possam contribuir para a melhoria de saúde da criança; incentivo ao aleitamento

\section{Referências}

1. Souza EC. Perfil etimológico das diarréias agudas em crianças atendidas em um pronto socorro de hospital regional da cidade de São Paulo. [dissertação de mestrado] São Paulo (SP): Faculdade de Medicina/USP; 2000.

2. Manual de Monitorização da Doença Diarréica Aguda- MDDA/ DDTHA/ CVE-SES/SP. São Paulo (SP); 2002.

3. Sack RB. Epidemiologia da diarréia aguda infecciosa. Resumos apresentados noSeminário Nestlé Nutrition. Filipinas: Nestlé Nutrition Services; 1996. p. 38.

4. Benguigui Y, et al. Ações de saúde materno-infantil à nível local: segundo as metas das cúpulas mundial em favor da infância. Brasília (DF): Ministério da Saúde; 1997. materno exclusivo e outras medidas reconhecidas de valor na redução da morbimortalidade infantil, como a terapia de reidratação oral (TRO); imunização e tratamento imediato da DDA, através da busca do serviço de saúde; acompanhamento periódico do crescimento e desenvolvimento das crianças; tratamento das intercorrências e vigilância do estado nutricional; desencadeamento e busca dos faltosos; realização de visitas domiciliares; e humanização do atendimento.

5. Ministério da Saúde. (BR) Secretaria de Assistência e Saúde. Coordenação de Saúde Materno Infantil. Assistência e controle da diarréia e cólera em serviços de saúde. Brasília (DF); 2002. p. 44. 6. Vranjac A. Diarréia e rotavírus. Rev Saude Publica 2004; 38(6) 7. Secretaria Municipal de Saúde de Ananindeua (PA). Sistema de Informação de Atenção Básica; 1999.

8. Marcondes E. Pediatria básica. $9^{a}$ ed. São Paulo (SP): Sarvier; 2002. 2v.

9. Beaglehole R, Bonita R, Kjellström T. Epidemiologia básica. São Paulo (SP): Santos; 1996. p.176.

10. Silva RCA. Qualidade da água: manancial subterrâneo em áreas urbanas de Feira de Santana (BA). Rev Saude Publica 2003; 8(4) 